\title{
Photolithography-free Skin-on-a-chip for Parallel Permeation Assays
}

\author{
Naoki Sasaki, Kimiaki Tsuchiya, and Hironori Kobayashi \\ Department of Applied Chemistry, Faculty of Science and Engineering, Toyo University, \\ 2100 Kujirai, Kawagoe, Saitama 350-8585 Japan
}

(Received September 3, 2018; accepted October 5, 2018)

Keywords: microfluidic, dermatitis, permeation, porous membrane, keratinocyte

Dermatitis (skin inflammation) is becoming a serious health problem that lowers the quality of life. Microfluidic devices have been employed as an alternative to conventional in vitro skin models utilized to evaluate irritants, allergens, or drug candidates for dermatitis. However, the following should be achieved: high-throughput analysis by parallelization, simple, easy, and inexpensive device fabrication, and real-time microscopic imaging. In this paper, a photolithography-free skin-on-a-chip for parallel permeation assays is presented. In this study, a consumer laser cutter is employed to form masters for substrates possessing branched microchannel patterns. A porous membrane is sandwiched between the substrates to form 24 crossings of the microchannels on a single device. HaCaT, a human skin keratinocyte cell line, is cultured until confluence on the membrane in the microchannels. Parallel permeation assays are demonstrated using fluorescent-dye-labelled dextran as a tracer and potassium dichromate as an irritant. We think that our technique will expand the use of skin-on-a-chip technology among biologists and pharmacologists, and will open up a new field of biosensing, drug development, and so on.

\section{Introduction}

The skin is the largest organ and covers the human body with barrier functions. It prevents both the leakage of internal constituents and heat from the body, and the penetration of foreign substances into the body, such as irritants and allergens. ${ }^{(1)}$ The disruption of these barrier functions leads to dermatitis (skin inflammation). ${ }^{(2)}$ Irritant contact dermatitis (ICD) is caused by the direct contact of the skin with irritants including detergents, shampoo, and so on, and represents ca. $80 \%$ of occupational contact dermatitis cases. ${ }^{(3)}$ Allergic contact dermatitis (ACD) is caused by the first contact of individuals with allergens and the subsequent direct contact of the skin with the allergen. Various types of materials can become allergens. Furthermore, it depends on the nature of individuals as to which materials become allergens. As a whole, both ICD and ACD lower the quality of life and are becoming serious health problems.

*Corresponding author: e-mail: nsasaki@toyo.jp https://doi.org/10.18494/SAM.2019.2125 
Various types of experimental systems have been utilized to evaluate irritants, allergens, or drug candidates for dermatitis. Animal tests are the most popular in vivo technique, but they suffer from ethical issues, high cost, and long analysis time. Franz diffusion cells enable the evaluation of analyte permeation against animal skin in vitro. ${ }^{(4)}$ Another in vitro skin model is based on reconstituted human epidermis and utilized to evaluate drug candidates. ${ }^{(5)}$ These in vitro systems enable evaluation under in vivo-like conditions. However, they require a large sample/reagent volume and have a low throughput.

Microfluidic devices have been employed as an alternative to in vitro skin models and offer reduced sample/reagent consumption. ${ }^{(6)}$ Various microfluidic skin models (or skin-ona-chip) have been reported. ${ }^{(7-10)}$ However, the following issues should be addressed: (i) Highthroughput analysis by parallelization is indispensable. Typical skin-on-a-chip experiments range from a week to a month. (ii) The method of fabricating the skin-on-a-chip should be simple, easy, and inexpensive. The use of microfabrication techniques for semiconductors (e.g., photolithography) is a barrier for researchers not familiar with microfluidics. (iii) Real-time and microscopy imaging-based analysis is suitable for detecting changes associated with dermatitis and analyzing multiple samples in parallel.

In this paper, we propose a photolithography-free skin-on-a-chip for parallel permeation assays. The concept of this technology is shown in Fig. 1. A porous membrane is sandwiched between branched microchannel patterns. As described later, the microchannel pattern can be easily fabricated without using photolithography. A number of microchannel crossings are arranged on a single device so that the permeation of analytes through a layer of keratinocytes cultured on the membrane can be assayed in parallel. The permeation of fluorescent tracers through the cell layer is observed in real time under a fluorescence microscope. The observation of multiple crossings in a field of view enables parallel assays. By introducing irritants into the microchannel, the effects of stimulation by the irritants on the permeation can be evaluated.

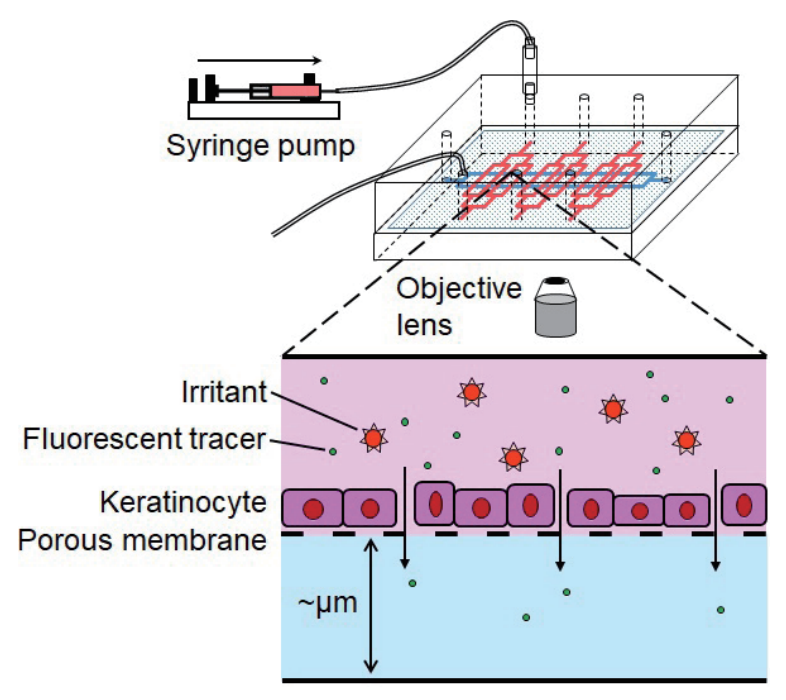

Fig. 1. (Color online) Concept of this technology. 


\section{Materials and Methods}

\subsection{Cells and culture procedures}

HaCaT (Cell Lines Service, Eppelheim, Germany) was selected as a representative human skin keratinocyte cell line. ${ }^{(11)} \mathrm{HaCaT}$ cells were cultured in a $12.5 \mathrm{~cm}^{2}$ cell culture flask (353018, Becton, Dickinson and Company, Franklin Lakes, NJ, USA). The cells were grown in Dulbecco's modified Eagle's medium (DMEM, D6429, Sigma-Aldrich, St. Louis, MO, USA) supplemented with 10\% fetal bovine serum (FBS, S1820-500, Biowest, Nuaillé, France) and $1 \times$ antibiotic-antimycotic (15240-062, Thermo Fisher Scientific, Waltham, MA, USA). 0.05\% ethylenediaminetetraacetic acid (EDTA) in phosphate-buffered saline (PBS) was prepared using EDTA (Wako Pure Chemical Industries, Osaka, Japan) and PBS (TAKARA BIO, Shiga, Japan).

Once cells reached $80-90 \%$ confluence, the medium in a cell culture flask was aspirated. The cells were rinsed with $4 \mathrm{~mL}$ of $0.05 \%$ EDTA in PBS. Then, $4 \mathrm{~mL}$ of fresh $0.05 \%$ EDTA in PBS was added, and the flask was incubated for 15 min at $37{ }^{\circ} \mathrm{C}$ under an atmosphere of $5 \% \mathrm{CO}_{2}$ and $95 \%$ air. Afterward, the solution in the flask was aspirated and the cells were incubated for 5 min with $3 \mathrm{~mL}$ of Hanks' balanced salt solution (HBSS(-), 17461-05, Nacalai Tesque, Kyoto, Japan) containing 0.05\% EDTA, 0.25\% trypsin (25-054-CI, Corning, Manassas, VA, USA), and $2 \%$ FBS. $3 \mathrm{~mL}$ of fresh medium was added to the flask and the obtained cell suspension was added to a $15 \mathrm{~mL}$ conical tube. The tube was centrifuged at $1300 \mathrm{rpm}$ for $5 \mathrm{~min}$ and the supernatant was aspirated. Finally, the cells were resuspended in the medium at the required concentration.

\subsection{Microfabrication}

A schematic illustration of the fabrication procedure of the microfluidic device is shown in Fig. 2(a). The top substrate possesses 3 microchannel patterns, each of which branches into 4 microchannels [Fig. 2(b)]. The bottom substrate possesses a branched microchannel pattern [Fig. 2(c)]. A porous membrane is sandwiched between the substrates. As a result, 24 crossings of the microchannels are formed. The design allows 24 parallel experiments under 3 different conditions on a single device. The design values of the microchannels are also shown in Figs. 2(b) and 2(c). We designed the microchannels so as to image a set of 8 crossings simultaneously with a $4 \times$ objective lens.

Microfluidic devices with porous membranes were fabricated as described previously ${ }^{(12)}$ with some modifications of the procedures of microchannel pattern formation. ${ }^{(13)}$ Briefly, bracketlike plates [Fig. 2(b)] were cut out of an acrylic sheet $(0.5 \mathrm{~mm}$ thickness, CLAREX, Nitto Jushi Kogyo, Tokyo, Japan) with a consumer laser cutter (HAJIME, Oh-Laser, Saitama, Japan). Three plates were glued to another acrylic plate $(1 \mathrm{~mm}$ thickness, CLAREX, Nitto Jushi Kogyo) at ca. $2 \mathrm{~mm}$ intervals to form a master for the top substrate. Plates with branched channel patterns [Fig. 2(c)] were cut out of an acrylic sheet $(0.3 \mathrm{~mm}$ thickness, CLAREX, Nitto Jushi Kogyo), and a master for the bottom substrate was fabricated in a similar manner. Poly(dimethylsiloxane) (PDMS) substrates possessing recessed channel patterns were fabricated 
as described previously. ${ }^{(12)}$ Through-holes for tubes were punched on the top substrate as shown in Fig. 2(a), using a biopsy punch (BPP-10F, Kai Industries, Gifu, Japan). Porous polyethylene terephthalate membranes with a pore size of $1 \mu \mathrm{m}$ and a thickness of ca. $12 \mu \mathrm{m}$ were cut from cell culture inserts (353102, Becton Dickinson and Company). Two through-holes were formed on the membrane, as shown in Fig. 2(a), using a soldering iron. Afterward, the membrane was sandwiched between the substrates possessing PDMS mortar layers as described previously. ${ }^{(12)}$ PTFE tubes (0.46 mm id, $0.92 \mathrm{~mm}$ od, Chukoh Chemical Industries, Tokyo, Japan) were glued to the through-holes at the end of the microchannels with the PDMS prepolymer.

\subsection{Cell viability assays}

The medium described in Sect. 2.1 was introduced into the top and bottom channel. The device was incubated overnight at $37{ }^{\circ} \mathrm{C}$ under an atmosphere of $5 \% \mathrm{CO}_{2}$ and $95 \%$ air. A suspension of $\mathrm{HaCaT}\left(3.6 \times 10^{6}\right.$ cells $\left.\mathrm{mL}^{-1}\right)$ was introduced manually into the top channel. The device was incubated in a similar manner for 5 days. Afterward, the channel was rinsed with $0.05 \%$

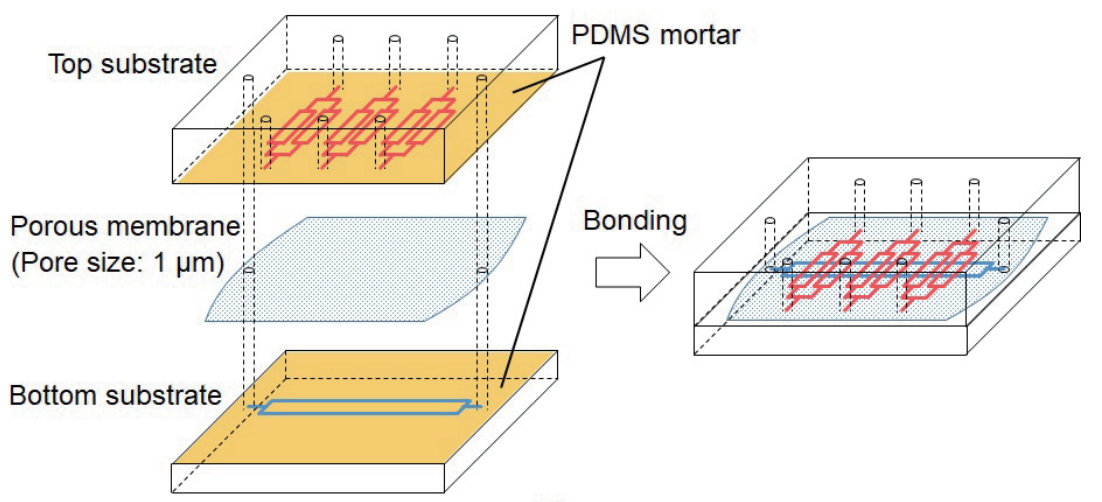

(a)

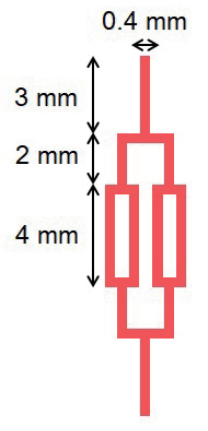

(b)

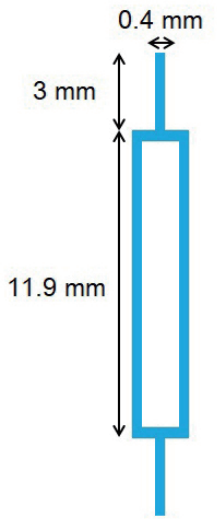

(c)

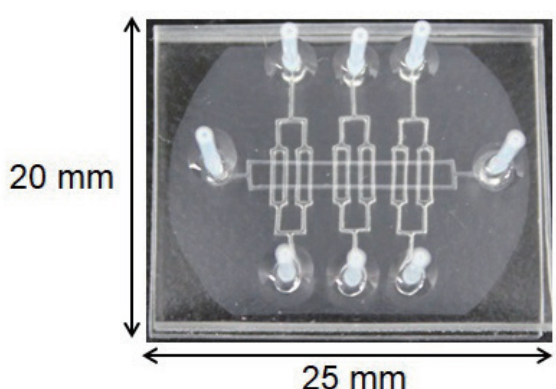

$25 \mathrm{~mm}$

(d)

Fig. 2. (Color online) (a) Schematic illustration of fabrication of the microfluidic device. Geometries of (b) top channel and (c) bottom channel. (d) Photograph of the microfluidic device. 
EDTA in PBS. A solution containing $2 \mu \mathrm{M}$ calcein AM and $4 \mu \mathrm{M}$ ethidium homodimer-1 (L3224, Thermo Fisher Scientific) was introduced into the channel and incubated for $30 \mathrm{~min}$.

Fluorescence and phase contrast images were acquired using a fluorescence microscope (IX71, Olympus, Tokyo, Japan) equipped with a CMOS camera (ORCA-Flash 4.0 V2, Hamamatsu Photonics, Hamamatsu, Japan), a $100 \mathrm{~W}$ high-pressure mercury lamp, and a 10× objective lens (NA 0.30). For live cell observation, a dichroic mirror block (U-MNIBA3, excitation 470-495 $\mathrm{nm}$ and emission 510-550 nm, Olympus) was used. For dead cell observation, another dichroic mirror block (U-MWIG3, excitation 530-550 nm and emission $>575 \mathrm{~nm}$, Olympus) was used. HCImage Live Software (Hamamatsu Photonics) and Image J 1.50i (National Institutes of Health, USA) were used to process the images.

\subsection{Permeation assays}

$\mathrm{HaCaT}$ cells were cultured as described in Sect. 2.3 for 15 days. The inlets of the microchannels were connected to a $1 \mathrm{~mL}$ syringe (1001TLL, Hamilton, Reno, NV, USA) via a bubble trap and a capillary. ${ }^{(14)}$ The top and bottom channels were flushed with a fresh medium. Then, another medium containing $10 \mu \mathrm{M}$ fluorescein isothiocyanate-dextran (FITC-Dex, average molecular weight 10000 , FD10S, Sigma-Aldrich) was introduced into the top channel using a syringe pump (model 210, KD Scientific, Holliston, MA, USA) at $10 \mu \mathrm{L} \mathrm{min}{ }^{-1}$. The microchannels were observed with a dichroic mirror block (U-MNIBA3) as described in Sect. 2.3. For the evaluation of the permeation, normalized fluorescence intensity (NFI) was defined as the ratio of the mean fluorescence intensity in the crossing at an indicated time to that in the top channel. Three crossings were randomly selected and analyzed.

Potassium dichromate $\left(\mathrm{K}_{2} \mathrm{Cr}_{2} \mathrm{O}_{7}\right)$ was selected as a model irritant because it is a strong oxidant as well as an allergen for dermatitis. ${ }^{(15)}$ A medium containing $100 \mu \mathrm{g} \mathrm{mL}{ }^{-1} \mathrm{~K}_{2} \mathrm{Cr}_{2} \mathrm{O}_{7}$ was introduced into both channels. The device was incubated for 2 nights and assayed again.

\section{Results and Discussion}

\subsection{Microfluidic device}

A photograph of the microfluidic device is shown in Fig. 2(d). The device was successfully fabricated without solution leakage or channel clogging. The channel width was reduced from 0.4 $\mathrm{mm}$ (designed width) to ca. $0.3 \mathrm{~mm}$ (actual width) because, in master fabrication, the laser beam with finite spot size traced the edges of the patterns shown in Figs. 2(b) and 2(c).

It should be noted that conventional membrane-integrated microfluidic devices require the sealing of the edges of the membrane to avoid solution leakage. ${ }^{(16)}$ In contrast, the present device does not require sealing because the channel patterns do not cross the edges. Through-holes on the membrane [see Fig. 2(a)] successfuly provided fluidic connections to the microchannel on the bottom substrate. 


\subsection{Cell culture}

A typical photograph of the crossing on the device is shown in Fig. 3. HaCaT cells were viable and confluent on the crossing. Several dead cells were observed in the microchannel, which indicates that the cells are stacked. We observed 12 crossings on 2 devices ( 24 crossings in total) and confirmed that $\mathrm{HaCaT}$ cells were confluent on all of the crossings. Therefore, the culture of $\mathrm{HaCaT}$ cells in parallel was demonstrated. Although the culture of $\mathrm{HaCaT}$ cells in three millimeter-sized compartments on a membrane-integrated microfluidic device has been reported, ${ }^{(8)}$ to the best of our knowledge, this is the first report on parallelization in a micrometer-sized space on a photolithography-free membrane-integrated microfluidic device.

\subsection{Permeation assays}

Typical photographs of the permeation assays are shown in Fig. 4. Just after the introduction of the FITC-Dex solution to the top channel, permeation was not observed [Fig. 4(b)]. However, after $15 \mathrm{~min}$ of the introduction, permeation was observed at 2 crossings [Fig. 4(c)]. After 30 min of the introduction, the amount of permeated FITC-Dex increased [Fig. 4(d)]. This result indicates that $\mathrm{HaCaT}$ cells were confluent at the other 6 crossings, but not at the 2 crossings. It is well known that contamination by air bubbles in a microchannel results in the removal of cells from channel surfaces. We think that the long culture period (15 days) may increase the possibility of the removal of cells. In total, permeation was observed at 2 crossings out of 24 crossings. Therefore, HaCaT cells were confluent at most of the crossings. Typical photographs of the permeation tests after stimulation with $\mathrm{K}_{2} \mathrm{Cr}_{2} \mathrm{O}_{7}$ are shown in Fig. 5. Permeation was clearly observed at all of the crossings in $15 \mathrm{~min}$.

The time course of NFI is shown in Fig. 6. NFI was almost constant before the stimulation, but increased to $\sim 1.4$ after the stimulation. Since the depths of the top and bottom channels were 0.5 and $0.3 \mathrm{~mm}$, respectively, the total depth at the crossings was $0.8 \mathrm{~mm}$. If FITC-Dex permeates in the membrane sufficiently and the concentration of FITC-Dex in the bottom channel is

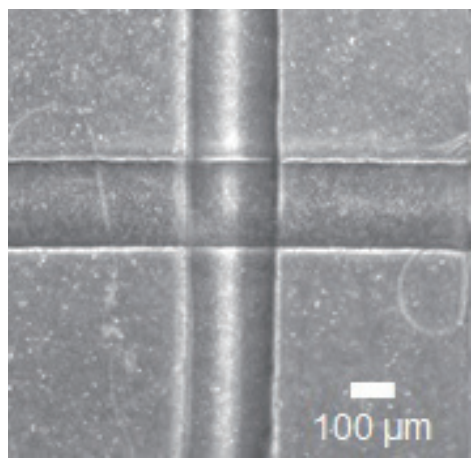

(a)

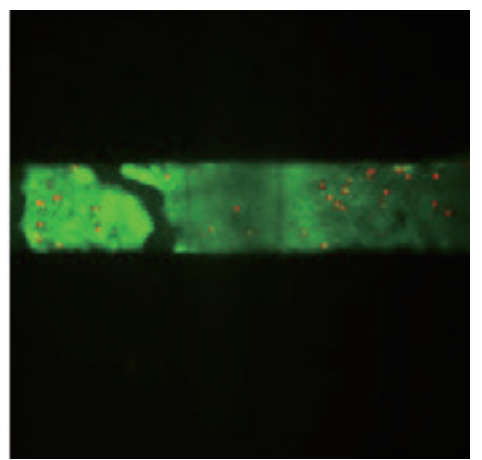

(b)

Fig. 3. (Color online) (a) Phase-contrast and (b) fluorescence images of HaCaT cells at a crossing in a microchannel. Green: live cells. Red: dead cells. 


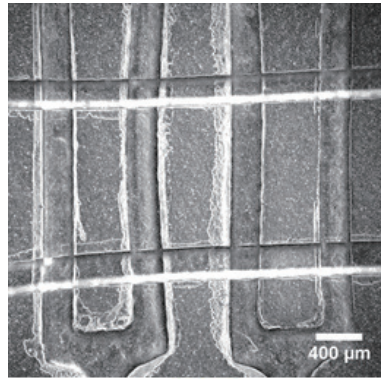

(a)

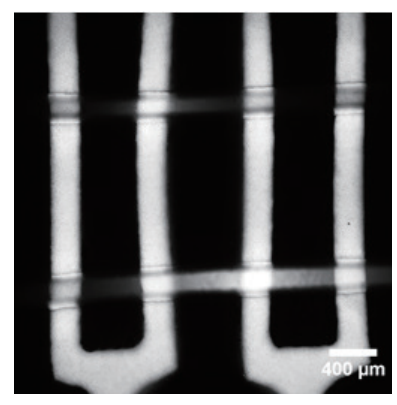

(c)

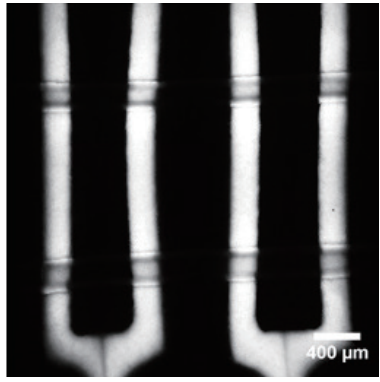

(b)

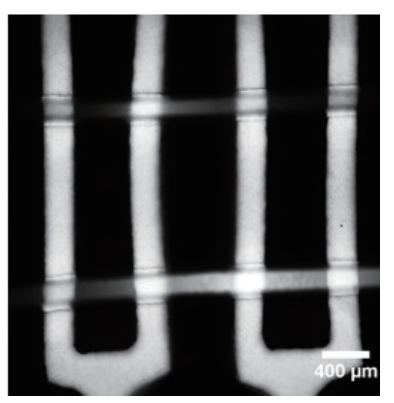

(d)

Fig. 4. Series of microscopy images of microchannels in permeation assays before stimulation. (a) Bright-field image showing the location of the microchannels. (b-d) Fluorescence images showing the permeation of FITCDex. The images were taken (b) immediately after the introduction of FITC-Dex solution, (c) 15 min after the introduction, and (d) 30 min after the introduction.

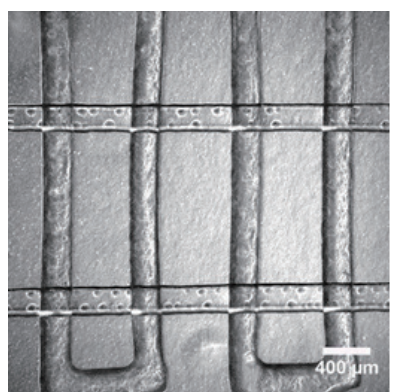

(a)

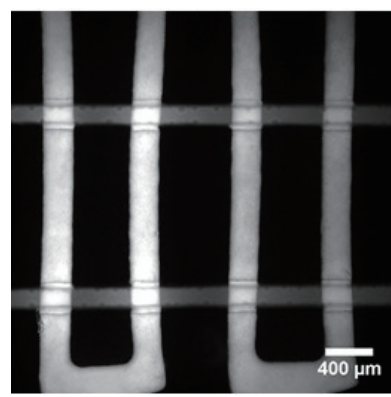

(c)

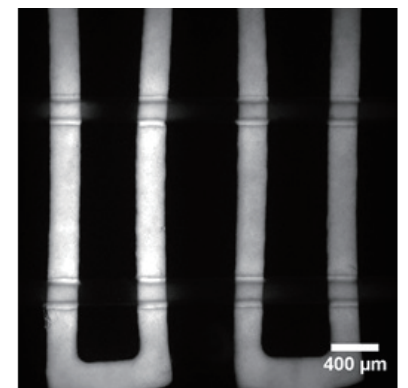

(b)

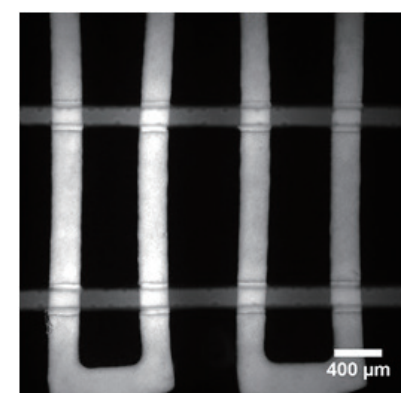

(d)

Fig. 5. Series of microscopy images of microchannels in permeation assays after stimulation with $\mathrm{K}_{2} \mathrm{Cr}_{2} \mathrm{O}_{7}$. (a) Bright-field image showing the location of the microchannels. (b-d) Fluorescence images showing the permeation of FITC-Dex. The images were taken (b) immediately after the introduction of FITC-Dex solution, (c) 15 min after the introduction, and (d) 30 min after the introduction. 


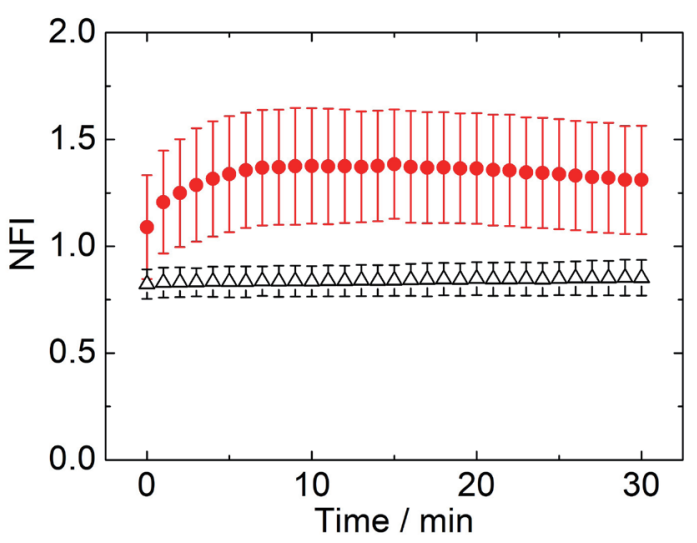

Fig. 6. (Color online) Time courses of NFI before (open triangles) and after (filled circles) stimulation with $\mathrm{K}_{2} \mathrm{Cr}_{2} \mathrm{O}_{7}$. Error bars indicate $\pm 1 \mathrm{SD}$ of three experiments.

the same as that of the top channel, NFI is expected to be 1.6, which is calculated as the ratio of the total depth at the crossings to that of the top channel. This value is almost the same as the experimentally obtained NFI. The statistical significance of the differences between the values obtained at 10 min was evaluated using the t-test, and the $p$-value was smaller than 0.05 . Therefore, it was demonstrated that the $\mathrm{HaCaT}$ cells were damaged during the stimulation with $\mathrm{K}_{2} \mathrm{Cr}_{2} \mathrm{O}_{7}$ and FITC-Dex permeated in the membrane. Since the time course of the permeation can be obtained by the assays, the present method can be applied to the evaluation of the kinetic parameters of permeation. Such an application will provide valuable information on, for example, the transdermal administration of drugs.

\section{Conclusions}

We have developed a photolithography-free skin-on-a-chip for parallel permeation assays. Microfluidic devices with a porous membrane and 24 microchannel crossings were successfully fabricated without using photolithography. HaCaT cells were confluent on all of the crossings. The permeation of fluorescent tracers through the HaCaT cell layer on the porous membrane was observed in parallel after the stimulation of the cells with $\mathrm{K}_{2} \mathrm{Cr}_{2} \mathrm{O}_{7}$. We think that our technique will expand the use of skin-on-a-chip technology among biologists and pharmacologists, and will open up a new field of biosensing, drug development, and so on.

\section{Acknowledgments}

The authors thank Professor Naoko Takahashi-Ando (Department of Applied Chemistry, Faculty of Science and Engineering, Toyo University) for her assistance with cell culture. This work was supported in part by The Science Research Promotion Fund. 


\section{References}

1 M. Yokouchi and A. Kubo: Exp. Dermatol. 27 (2018) 876. https://doi.org/doi:10.1111/exd.13742

2 I. Jakasa, J. P. Thyssen, and S. Kezic: Exp. Dermatol. 27 (2018) 909. https://doi.org/10.1111/exd.13704

3 C.-H. Tan, S. Rasool, and G. A. Johnston: Clin. Dermatol. 32 (2014) 116. https://doi.org/10.1016/ j.clindermatol.2013.05.033

4 A. Zeb, O. S. Qureshi, H. S. Kim, J. H. Cha, and J. K. Kim: Int. J. Nanomed. 11 (2016) 3813. https://doi. org/10.2147/ijn.s109565

5 D. M. Danilenko, G. D. L. Phillips, and D. Diaz: Toxicol. Pathol. 44 (2016) 555. https://doi. org $/ 10.1177 / 0192623316632074$

6 L. J. van den Broek, L. I. J. C. Bergers, C. M. A. Reijnders, and S. Gibbs: Stem Cell Rev. Rep. 13 (2017) 418. https://doi.org/10.1007/s12015-017-9737-1

7 H. E. Abaci, K. Gledhill, Z. Guo, A. M. Christiano, and M. L. Shuler: Lab Chip 15 (2015) 882. https://doi. org/10.1039/c4lc00999a

8 Q. Ramadan and F. C. W. Ting: Lab Chip 16 (2016) 1899. https://doi.org/10.1039/c6lc00229c

9 N. Mori, Y. Morimoto, and S. Takeuchi: Biomaterials 116 (2017) 48. https://doi.org/10.1016/ j.biomaterials.2016.11.031

10 M. Alberti, Y. Dancik, G. Sriram, B. Wu, Y. L. Teo, Z. Feng, M. Bigliardi-Qi, R. G. Wu, Z. P. Wang, and P. L. Bigliardi: Lab Chip 17 (2017) 1625. https://doi.org/10.1039/c6lc01574c

11 P. Boukamp, R. T. Petrussevska, D. Breitkreutz, J. Hornung, A. Markham, and N. E. Fusenig: J. Cell. Biol. 106 (1988) 761. https://doi.org/10.1083/jcb.106.3.761

12 N. Sasaki, M. Tatanou, T. Suzuki, Y. Anraku, A. Kishimura, K. Kataoka, and K. Sato: Anal. Sci. 32 (2016) 1307. https://doi.org/10.2116/analsci.32.1307

13 N. Sasaki, T. Hayashi, N. Inoue, and M. Onishi: Bunseki Kagaku 67 (2018) 379. https://doi.org/10.2116/ bunsekikagaku.67.379

14 N. Sasaki, M. Shinjo, S. Hirakawa, M. Nishinaka, Y. Tanaka, K. Mawatari, T. Kitamori, and K. Sato: Electrophoresis 33 (2012) 1729. https://doi.org/10.1002/elps.201100691

15 M. Bock, A. Schmidt, T. Bruckner, and T. L. Diepgen: Br. J. Dermatol. 149 (2003) 1165. https://doi.org/10.1111/ j.1365-2133.2003.05748.x

16 R. F. Ismagilov, J. M. K. Ng, P. J. A. Kenis, and G. M. Whitesides: Anal. Chem. 73 (2001) 5207. https://doi. org/10.1021/ac010502a 